\title{
Susceptibility Spectroscopy in FeNiSiB Microwires
}

\author{
J. Olivera ${ }^{a, b}$, J. Gamcova ${ }^{a}$, R. VArgA ${ }^{a}$, J.D. SAntos ${ }^{b}$, \\ V.M. PRIDA ${ }^{b}$, M.L. SANCHEZ ${ }^{b}$, B. HERnANDO ${ }^{b}$ AND A. ZhUKOV ${ }^{c}$ \\ ${ }^{a}$ Inst. Phys., Fac. Sci., UPJS, Park Angelinum 9, 04154 Kosice, Slovakia \\ ${ }^{b}$ Dept. Fisica, Universidad de Oviedo, Calvo Sotelo s/n, 33007-Oviedo, Spain \\ ${ }^{c}$ Dpto. Física de Materiales, Fac. Química, UPV/EHU \\ 1072, 20080, San Sebastián, Spain
}

\begin{abstract}
Here, we present the study on the influence of the $\mathrm{Ni}$ content on the magnetization processes of the amorphous glass-coated $\mathrm{Fe}_{77.5-x} \mathrm{Ni}_{x} \mathrm{Si}_{7.5} \mathrm{~B}_{15}$ $(x=0-40)$ microwires. Measurement of the amplitude dependence of the complex susceptibility was used to observe the magnetization process. It is shown that the magnetization process in the presented samples runs mainly through the domain wall motion.
\end{abstract}

PACS numbers: 75.50.Kj, 75.60.Jk

\section{Introduction}

Amorphous magnetic microwires are novel materials, which are characterized by unusual soft magnetic properties, such as magnetic bistability and Giant MagnetoImpedance (GMI) effect [1].

The magnetic behavior of this microwire strongly depends on their composition, which is directly responsible of the sign and magnitude of magnetostriction constant, and also on the values and distribution of internal stresses induced during preparation [2].

The magnetic properties of amorphous microwires are usually described in terms of the core-shell model [3]. In accordance with the model, a microwire with positive magnetostriction consists of one large axial domain surrounded by the outer domains with radially oriented magnetization.

Measurement of the amplitude dependence of the complex susceptibility is a useful method for studying dynamic magnetization processes in ferromagnetic materials because the magnetization processes can be resolved $[4,5]$. We apply this method here to study the magnetization process in amorphous glass-coated $\mathrm{FeNiSiB}$ microwires with different $\mathrm{Fe} / \mathrm{Ni}$ ratios. 


\section{Experimental}

Amorphous glass-coated microwires of composition $\mathrm{Fe}_{100-x} \mathrm{Ni}_{x} \mathrm{Si}_{7.5} \mathrm{~B}_{15}(x=$ 0-40), with internal diameter $15 \mu \mathrm{m}$ and total diameter $33 \mu \mathrm{m}$ were produced by the Taylor-Ulitovski method. The length of all samples was $5 \mathrm{~cm}$. The amplitude dependence of the complex susceptibility was measured by the lock-in amplifier at $120 \mathrm{~Hz}$ at room temperature.

\section{Results and discussion}

In order to analyze the type of magnetization processes, the field dependence of both components of the complex susceptibility are represented in Fig. 1 for four different concentrations of $\mathrm{Ni}$. All dependences are characterized by the similar

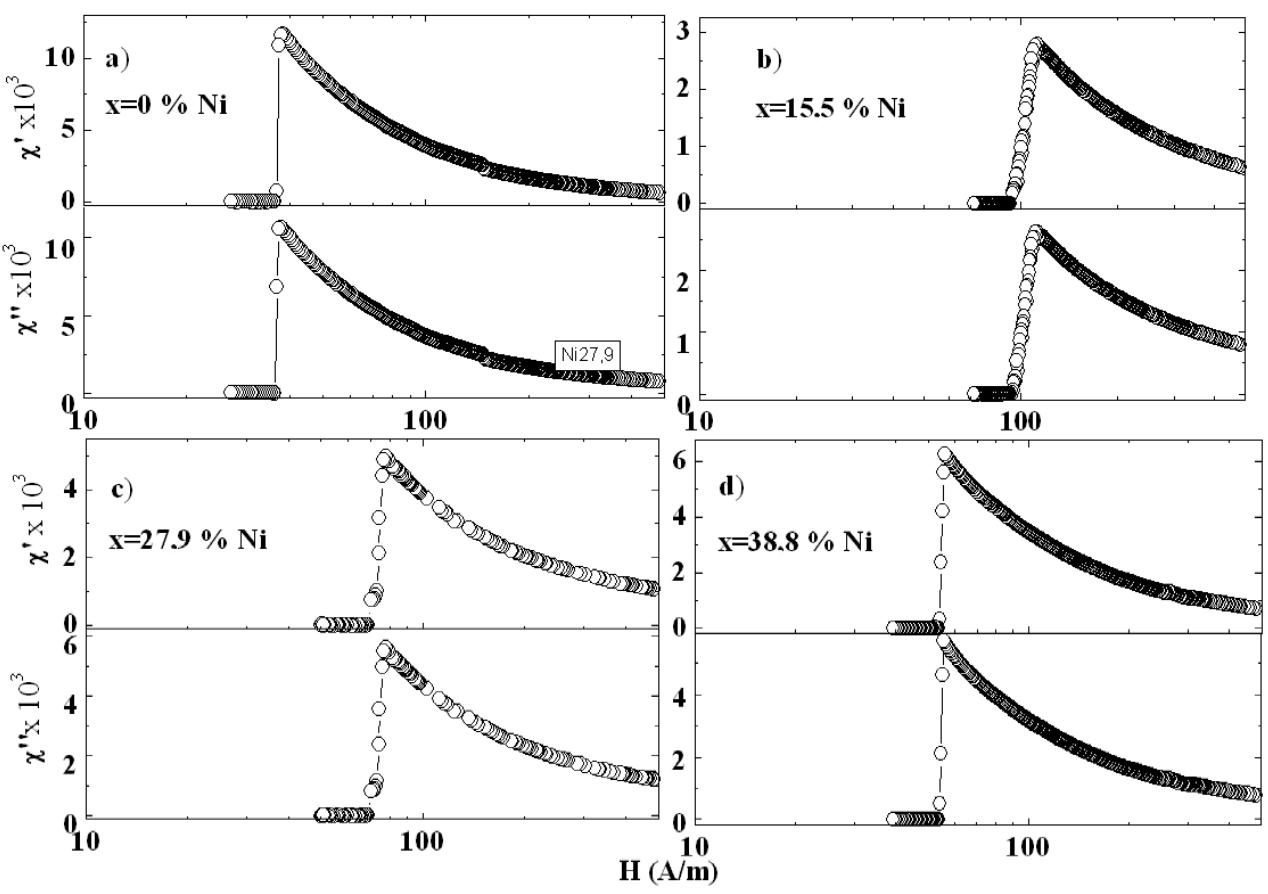

Fig. 1. Amplitude dependences of the real and imaginary part of the complex susceptibility in the amorphous glass-coated $\mathrm{Fe}_{77.5-x} \mathrm{Ni}_{x} \mathrm{Si}_{7.5} \mathrm{~B}_{15}$ microwire.

forms of both components (real as well as imaginary one). The susceptibility is zero up to the switching field $H_{\mathrm{sw}}$, where it abruptly increases as a result of the depining of the closure domain wall, according to the expected behavior in wires with positive magnetostriction [6]. Above the $H_{\mathrm{sw}}$, the imaginary parts immediately decrease in the hyperbolic dependence, whereas in the real part small concave decrease shows the reversible moment rotation in the external domain structure with the radial magnetization [5]. Above the switching field, the sample 
is technically saturated and both susceptibility components decrease hyperbolically with the applied field. Such behavior is typical of the sample characterized by the single Barkhausen jump [5].

In contrary, samples with a composition of $15.5 \%$ and $27.9 \% \mathrm{Ni}$ (Fig. 1b,c) are characterized by a more complicated magnetization process. Although both parts show similar dependence on the amplitude of the applied magnetic field (which points to the domain wall motion), the susceptibility increases weakly in contrary to the samples with $x=0$ and 38.8 at.\% of Ni. These samples show higher axial switching field, which is probably comparable to the anisotropy field of the radial domain structure. Therefore, complex domain wall movements are observed instead of the single Barkhausen jump.

The switching field and the maximum value of susceptibility are shown in Fig. 2 as a function of at.\% Ni. The switching field depends strongly on the values of the effective anisotropy, saturation magnetostriction, and saturation magnetization [7], being modified by means of substitution of $\mathrm{Ni}$ for Fe. However, the substitution of $\mathrm{Fe}$ by $\mathrm{Ni}$ in amorphous materials does not result in the monotonous change of the above-described parameters [8]. Therefore, two maxima appear (at $x=15$ and 35 at. $\% \mathrm{Ni}$ ) in the compositional dependence of the switching field of amorphous FeNiSiB microwires. On the other hand, susceptibility is inversely proportional to the switching field, showing two local minima at the same position.

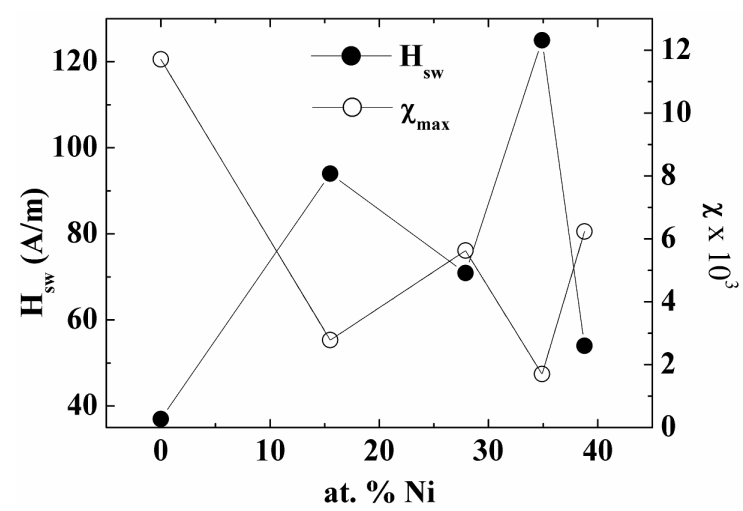

Fig. 2. Dependence of switching field and maximum susceptibility on the Ni content of $\mathrm{Fe}_{100-x} \mathrm{Ni}_{x} \mathrm{Si}_{7.5} \mathrm{~B}_{15}(x=0-40)$ microwires.

\section{Conclusion}

The magnetization process in amorphous glass-coated $\mathrm{Fe}_{100-x} \mathrm{Ni}_{x} \mathrm{Si}_{7.5} \mathrm{~B}_{15}$ $(x=0-40)$ has been studied by means of amplitude dependence of complex susceptibility. It was shown that the magnetization process in the amorphous microwires with positive magnetostriction is mainly due to the domain wall movement. 
The substitution of $\mathrm{Ni}$ for Fe creates new magnetostrictive interactions and this new short-range order affects the effective anisotropy and magnetostriction. This way modifying the magnetostriction and effective anisotropy one can obtain different magnetization processes and magnetic structures. The maximum of switching field was observed for the samples with 15 and 35 at.\% of Ni, which are characterized by the complex domain wall movement. In order to clarify this behavior, studies of the amplitude dependence of susceptibility at different temperature are being carried out in those microwires.

\section{Acknowledgments}

J.O. acknowledges the support from MEC. This work was supported by the scientific grants APVT-20-007804, VEGA 1/3035/06 and MAT2006-13925-C02-01.

\section{References}

[1] A. Zhukov, J. Gonzalez, J.M. Blanco, M. Vazquez, V. Larin, J. Mater. Res. 15, 2197 (2000).

[2] H. Chiriac, T.A. Ovari, Gh. Pop, Phys. Rev. B 52, 10104 (1995).

[3] M. Vazquez, A. Hernando, J. Phys. D, Appl. Phys. 29, 939 (1996).

[4] S.S. Yoon, C.G. Kim, Appl. Phys. Lett. 78, 3280 (2001).

[5] M. Ipatov, R. Varga, A. Zhukov, J.M. Blanco, J. Gonzalez, V. Zhukova, J. Non-Crystal. Solids 353, 928 (2007).

[6] K. Mohri, F.B. Humphrez, K. Kawashima, K. Kimura, M. Muzutani, IEEE Trans. Magn. 26, 1789 (1990).

[7] R. Varga, K.L. Garcia, A. Zhukov, M. Vazquez, P. Vojtanik, Appl. Phys. Lett. 83, 2620 (2003).

[8] P. Vojtanik, E. Komova, P. Petrovic, R. Varga, H. Sassik, R. Grossinger, Acta Phys. Slov. 48, 719 (1999). 